\title{
Comparison of the Kato-Katz method and ether-concentration technique for the diagnosis of soil-transmitted helminth infections in the framework of a randomised controlled trial
}

\author{
B. Speich • J. Utzinger • H. Marti - S. M. Ame • S. M. Ali • \\ M. Albonico • J. Keiser
}

Received: 20 September 2013 / Accepted: 8 November 2013 /Published online: 23 November 2013

(C) Springer-Verlag Berlin Heidelberg 2013

\begin{abstract}
Soil-transmitted helminth infections are a major public health problem. An accurate diagnosis is important in order to identify individuals and communities in need of intervention, and for monitoring drug efficacy and potential emergence of resistance. We compared the accuracy of the Kato-Katz method and ether-concentration technique for the diagnosis of soil-transmitted helminth infections within a randomised controlled trial. Quadruplicate Kato-Katz thick smears (duplicate Kato-Katz from two stool samples each) were examined before (baseline) and 3 weeks after treatment (follow-up). Additionally, at baseline and follow-up, the first stool sample was subjected to an ether-concentration method. We determined the prevalence, sensitivity, negative predictive value, diagnostic agreement and cure rates for single and duplicate Kato-Katz thick smears from the first stool sample, quadruplicate Kato-Katz thick smears produced from two
\end{abstract}

\footnotetext{
B. Speich · J. Keiser $(\square)$

Department of Medical Parasitology and Infection Biology, Swiss

Tropical and Public Health Institute, P.O. Box, CH-4002 Basel,

Switzerland

e-mail: jennifer.keiser@unibas.ch
}

B. Speich $\cdot$ J. Utzinger $\cdot$ H. Marti $\cdot$ J. Keiser

University of Basel, Basel, Switzerland

\section{J. Utzinger}

Department of Epidemiology and Public Health, Swiss Tropical and Public Health Institute, Basel, Switzerland

\section{H. Marti}

Department of Medical and Diagnostic Services, Swiss Tropical and Public Health Institute, Basel, Switzerland

S. M. Ame $\cdot$ S. M. Ali

Public Health Laboratory (Pemba) - Ivo de Carneri, Chake Chake, Tanzania

\section{Albonico}

Ivo de Carneri Foundation, Milano, Italy stool samples and single ether-concentration as compared to our 'gold' standard (i.e. quadruplicate Kato-Katz plus etherconcentration). Quadruplicate Kato-Katz revealed a higher sensitivity than single ether-concentration for Trichuris trichiura at baseline ( $94.3 \%$ vs. $88.5 \%, p=0.002)$ and follow-up $(93.8 \%$ vs. $83.5 \%, p<0.001)$. In contrary, at follow-up, etherconcentration showed a higher sensitivity than quadruplicate Kato-Katz for Ascaris lumbricoides diagnosis $(86.7 \%$ vs. $46.7 \%, p=0.012$ ). The ether-concentration method showed similar or slightly higher sensitivity than the Kato-Katz technique based on a single stool sample for all soil-transmitted helminth infections. The estimated cure rates were heavily dependent on the diagnostic technique and sampling effort. In conclusion, data on the prevalence of soil-transmitted helminth infections and the efficacy of anthelminthics are greatly influenced by the diagnostic method and sampling effort. The etherconcentration technique is a valuable alternative to the KatoKatz method for helminth diagnosis.

\section{Introduction}

Chronic infections with one or several of the common soiltransmitted helminths (i.e. Ascaris lumbricoides, Trichuris trichiura and hookworm) cause an estimated global burden of 5.2 million disability-adjusted life years (DALYs) [1]. Preventive chemotherapy, that is, the large-scale administration of anthelminthic drugs (mainly the two benzimidazoles albendazole and mebendazole) to at-risk populations to avert morbidity, is the mainstay of control [2-4]. Accurate diagnostic tools are of pivotal importance for the identification of infected individuals, assessing endemicity in a given epidemiological setting and monitoring of drug efficacy and response to interventions, including resistance development $[5,6]$. The most widely used technique to diagnose intestinal helminth infections (i.e. soil-transmitted helminths, Schistosoma 
japonicum, S. mansoni and S. mekongi) is the Kato-Katz method [7-11]. A single Kato-Katz thick smear examines, on average, $41.7 \mathrm{mg}$ of stool on a microscopic slide for the detection and quantification of helminth eggs. The diagnostic sensitivity of the Kato-Katz method can be improved by examining multiple thick smears from a single stool sample or by examining multiple stool samples [8, 12-17]. Nevertheless, the Kato-Katz technique has limitations, in particular, when the prevalence of soil-transmitted helminth infections is lower than $20 \%$ or when infection intensities are low $[5,18]$.

The ether-concentration method is a diagnostic approach which can detect soil-transmitted helminth eggs within a large amount of a fixed stool sample [19]. For example, a recent study conducted in the People's Republic of China revealed that the ether-concentration method based on one single stool sample is nearly as sensitive as the Kato-Katz method based on two to three stool samples for the diagnosis of soiltransmitted helminth infections [14].

The aim of the present study was to assess the sensitivity of single and multiple Kato-Katz thick smears in comparison to the ether-concentration method for the diagnosis of soiltransmitted helminths before and after a treatment intervention. Stool samples were collected in the frame of a randomised controlled trial, carried out among school-aged children on Pemba Island, Tanzania, which evaluated the efficacy and safety of nitazoxanide, albendazole and nitazoxanide-albendazole against $T$. trichiura infections [20]. We assessed the influence of the diagnostic technique and sampling effort on the prevalence of soil-transmitted helminth infections before and after treatment, as well as on observed cure rates (CRs).

\section{Materials and methods}

\section{Ethics statement}

The data presented here stem from a randomised controlled trial carried out among school-aged children on Pemba Island, Tanzania. The ethics statement has been presented previously [20]. In short, ethical clearance was obtained from the Ministry of Health and Social Welfare in Zanzibar (ZAMREC, reference no. 0001/010) and from the ethics committee of Basel, Switzerland (EKBB, reference no. 225/10). The study is registered at Current Controlled Trials (ISRCTN08336605). Written informed consent was acquired from the children's parents or legal guardians to participate in this trial. Children provided assent. At the end of the study, all children remaining positive for soil-transmitted helminths were offered standard treatment (i.e. $400 \mathrm{mg}$ albendazole).
Collection and processing of stool samples

Stool samples were collected from school-aged children participating in the trial, which assessed the safety and efficacy of the following four treatments: (i) nitazoxanide-albendazole combination (1,000 mg-400 mg), with each drug given separately on two consecutive days; (ii) single albendazole (400 mg); (iii) single nitazoxanide (1,000 mg); and (iv) placebo. The clinical trial took place from June to July 2011. The study setting and trial procedures have been described elsewhere [20].

Two stool samples collected before (baseline) and 3 weeks after treatment (follow-up) were available from 657 children. From each stool sample, duplicate Kato-Katz thick smears were prepared according to guidelines put forward by the World Health Organization (WHO) [18]. Within $30 \mathrm{~min}$, Kato-Katz thick smears were examined quantitatively for hookworm eggs. Subsequently, the slides were re-read for A. lumbricoides and T. trichiura eggs that were counted and recorded separately. Additionally, from 550 children, approximately $2 \mathrm{~g}$ of stool from the first specimen before and after treatment were fixed in $10 \mathrm{ml}$ of $5 \%$ formalin in $15-\mathrm{ml}$ Falcon tubes. Formalin-fixed samples were transferred to the Swiss Tropical and Public Health Institute (Swiss TPH) in Basel, Switzerland, and semi-quantitatively analysed for soiltransmitted helminth eggs and intestinal protozoan cysts within 10 months, using an ether-concentration method [21, 22]. Results pertaining to intestinal protozoa infections have been described elsewhere [23]. Complete datasets were available from 528 individuals (i.e. quadruplicate Kato-Katz thick smears before and after treatment, as well as a single formalin-fixed stool sample subjected to an etherconcentration method before and after treatment) and considered for the present work.

Statistical analysis

All data were double-entered into an Excel file (Microsoft 2010) and cross-checked. For statistical analysis, Stata version 10.1 was used (StataCorp., College Station, TX, USA).

The prevalence at baseline and treatment follow-up was calculated as the percentage of individuals diagnosed positive for a specific soil-transmitted helminth species, considering the following diagnostic approaches: (i) the first Kato-Katz thick smear from the first stool sample; (ii) duplicate KatoKatz thick smears from the first stool sample; (iii) quadruplicate Kato-Katz thick smears (two stool samples, each subjected to duplicate Kato-Katz); and (iv) single ether-concentration test from the first stool sample. As the diagnostic 'gold' standard, we considered the combined results from quadruplicate Kato-Katz thick smears plus single ether-concentration. Each sample found positive with either method was considered as "true positive". The sensitivity and negative predictive value (NPV) were calculated for the different diagnostic 
approaches and sampling efforts before and after treatment, assuming that the 'gold' standard had a sensitivity of $100 \%$. Differences in prevalence were determined under the assumption that non-overlapping $95 \%$ confidence intervals (CIs) indicate statistical significance. Cohen's kappa measure was used to assess agreement between the two methods and between different sampling efforts for the Kato-Katz technique, as follows: $\mathrm{K}<0$, no agreement; $\mathrm{K}=0-0.20$, poor agreement; $\kappa=0.21-0.40$, fair agreement; $\kappa=0.41-0.60$, moderate agreement; $\mathrm{K}=0.61-0.80$, substantial agreement; and $\mathrm{\kappa}=0.81-1.00$, nearly perfect agreement $[24,25]$. The McNemar test was used to examine differences in sensitivity between diagnostic methods. Therefore, only individuals who were positive according to the 'gold' standard were included. For a sample size of 20 or fewer individuals in the discordant pair, the exact McNemar value was taken $\left(p^{*}\right)$, otherwise, we used the McNemar $\chi^{2}$ value ( $p$ ) [26]

The CR was calculated as the percentage of individuals who were diagnosed negative at the 3 -week posttreatment follow-up, but had a positive diagnostic test before drug administration. CRs were calculated for each diagnostic approach and for each treatment arm individually. Differences between CRs among the two diagnostic methods and varying sampling efforts were compared with a two-sample test of proportion.

\section{Results}

Baseline data

The overall prevalence in school-aged children in the study setting before treatment based on our 'gold' standard was $87.1 \%$ (95\% CI, 84.3-90.0 \%) for T. trichiura, $11.2 \%$ (95\% CI, 8.5-13.9\%) for hookworm and $6.8 \%$ (95\% CI, 4.7-9.0 \%) for A. lumbricoides (Table 1). For all three soiltransmitted helminth species, statistically significantly lower prevalences were calculated when relying on the Kato-Katz method from a single stool sample compared to the 'gold' standard, regardless of whether stool samples were subjected to single or duplicate Kato-Katz thick smears.

Duplicate Kato-Katz thick smears identified a larger number of children with T. trichiura and A. lumbricoides infection compared to the ether-concentration method, while the etherconcentration method detected slightly more hookworm cases. However, these differences lacked statistical significance, as indicated by overlapping $95 \%$ CIs.

Our study revealed a high sensitivity for all diagnostic methods for the detection of T. trichiura eggs (Table 1). On the contrary, the sensitivity for hookworm and A. lumbricoides was considerably lower for all diagnostic methods. Quadruplicate Kato-Katz thick smears showed the highest sensitivity for T. trichiura and A. lumbricoides diagnosis compared to less intensive sampling and the etherconcentration method. The McNemar test revealed a statistically significantly higher sensitivity at baseline using quadruplicate Kato-Katz thick smears compared to the etherconcentration method for diagnosing T. trichiura $(94.3 \%$ vs. $88.5 \% ; p=0.002$; Table 2 ). The ether-concentration method had the highest sensitivity for hookworm. We observed a significant difference for hookworm diagnosis with the etherconcentration method compared to a single Kato-Katz (69.5\% vs. $39.0 \% ; p=0.004)$.

NPVs were high for both methods and different sampling efforts of the Kato-Katz technique for hookworm and A. lumbricoides diagnosis (92.9-98.2\%), while lower NPVs were observed for T. trichiura, ranging from $49.6 \%$ (single Kato-Katz) to $72.3 \%$ (quadruplicate Kato-Katz).

The agreement between the ether-concentration method and quadruplicate Kato-Katz thick smears was moderate for all helminth species before treatment ( $T$. trichiura, $\mathrm{K}=0.54$; A. lumbricoides, $\mathrm{K}=0.48$; hookworm, $\mathrm{K}=0.47$ ) (Table 2).

\section{Treatment follow-up}

The overall prevalence 3 weeks after treatment according to our 'gold' standard was $84.9 \%$ (95\% CI, 81.8-87.9\%) for T. trichiura, $7.2 \%$ (95 \% CI, 5.0-9.4\%) for hookworm and $5.7 \%$ (95\% CI, 3.7-7.7\%) for A. lumbricoides (Table 3). Single stool examination with the Kato-Katz and etherconcentration methods resulted in a significantly lower prevalence for T. trichiura compared to the 'gold' standard. Duplicate Kato-Katz thick smears revealed a significantly higher number of T. trichiura compared to the ether-concentration method. Duplicate Kato-Katz showed a better diagnostic performance than the ether-concentration method with regard to detecting hookworm eggs. On the other hand, the etherconcentration method detected more $A$. lumbricoides cases compared to quadruplicate Kato-Katz thick smears. However, these differences lacked statistical significance.

The McNemar test revealed that quadruplicate Kato-Katz thick smears were more sensitive for diagnosing T. trichiura than the ether-concentration method $(93.8 \%$ vs. $83.5 \%$; $p<$ 0.001; Table 2). On the other hand, the ether-concentration method showed a significantly higher sensitivity for A. lumbricoides compared to the Kato-Katz method based on one stool sample ( 86.7 vs. $40.0 ; p=0.003$ ), but also compared to quadruplicate Kato-Katz thick smears ( 86.7 vs. 46.7 ; $p=0.012$ ).

The NPVs calculated at the 3-week post-treatment followup for T. trichiura ranged from $55.2 \%$ (single Kato-Katz) to $74.1 \%$ (quadruplicate Kato-Katz), while the NPVs for hookworm and $A$. lumbricoides diagnosis were above $95 \%$ for both techniques, regardless of the sampling effort. The agreement between quadruplicate Kato-Katz thick smears and the ether-concentration method was moderate at follow-up 
Table 1 Prevalence of soil-transmitted helminth infections on Pemba Island, Tanzania, in mid-2011 according to different diagnostic methods and their corresponding sensitivities and negative predictive values at baseline

\begin{tabular}{|c|c|c|c|c|c|c|}
\hline \multirow[t]{2}{*}{ Parasite } & \multirow[t]{2}{*}{ Diagnostic technique } & \multicolumn{3}{|c|}{ Number of infected children } & \multirow{2}{*}{$\begin{array}{l}\text { Sensitivity in \% } \\
(95 \% \mathrm{CI})\end{array}$} & \multirow{2}{*}{$\begin{array}{l}\text { NPV } \\
(95 \% \mathrm{CI})\end{array}$} \\
\hline & & $n$ & $\%$ & $95 \% \mathrm{CI}$ & & \\
\hline \multirow[t]{5}{*}{ T. trichiura } & 'Gold' standard & 460 & 87.1 & $84.3-90.0$ & 100.0 & 100.0 \\
\hline & Single Kato-Katz & 391 & 74.1 & $70.3-77.8$ & $85.0(81.4-88.1)$ & $49.6(41.0-58.3)$ \\
\hline & Duplicate Kato-Katz & 406 & 76.9 & $74.4-80.5$ & $88.3(85.0-91.1)$ & $55.7(46.5-64.6)$ \\
\hline & Quadruplicate Kato-Katz & 434 & 82.2 & $78.9-85.5$ & $94.3(91.8-96.3)$ & $72.3(62.0-80.8)$ \\
\hline & Ether-concentration & 407 & 77.1 & $73.5-80.7$ & $88.5(85.2-91.2)$ & $56.2(46.9-65.1)$ \\
\hline \multirow[t]{5}{*}{ Hookworm } & 'Gold' standard & 59 & 11.2 & $8.5-13.9$ & 100.0 & 100.0 \\
\hline & Single Kato-Katz & 23 & 4.4 & $2.6-6.1$ & $39.0(26.5-52.6)$ & $92.9(90.2-94.9)$ \\
\hline & Duplicate Kato-Katz & 24 & 4.6 & $2.8-6.3$ & $40.7(28.1-54.3)$ & $93.1(90.4-95.0)$ \\
\hline & Quadruplicate Kato-Katz & 38 & 7.2 & $5.0-9.4$ & $64.4(50.9-76.4)$ & $95.7(93.4-97.3)$ \\
\hline & Ether-concentration & 41 & 7.8 & $5.5-10.1$ & $69.5(56.1-80.8)$ & $96.3(94.1-97.7)$ \\
\hline \multirow[t]{5}{*}{ A. lumbricoides } & 'Gold' standard & 36 & 6.8 & $4.7-9.0$ & 100.0 & 100.0 \\
\hline & Single Kato-Katz & 14 & 2.7 & $1.3-4.0$ & $38.9(23.1-56.5)$ & $95.7(93.5-97.2)$ \\
\hline & Duplicate Kato-Katz & 16 & 3.0 & $1.6-4.5$ & $44.4(27.9-61.9)$ & $96.1(93.9-97.5)$ \\
\hline & Quadruplicate Kato-Katz & 27 & 5.1 & $3.2-7.0$ & $75.0(57.8-87.9)$ & $98.2(96.5-99.1)$ \\
\hline & Ether-concentration & 21 & 4.0 & $2.3-5.7$ & $58.3(40.8-74.5)$ & $97.0(95.1-98.3)$ \\
\hline
\end{tabular}

(T. trichiura, $\mathrm{\kappa}=0.49 ;$ A. lumbricoides, $\mathrm{\kappa}=0.48$; hookworm, $\mathrm{K}=0.42)($ Table 2).

\section{Estimated CRs}

CRs calculated for each diagnostic approach for the three soiltransmitted helminths, stratified by treatment arm, are presented in Table 4. According to our 'gold' standard, CRs for T. trichiura were $11.7 \%$ (95\% CI, 5.3-18.0\%) for the nitazoxanide-albendazole combination, $9.1 \%$ (95\% CI, 3.6$14.5 \%$ ) for single albendazole and $1.6 \%$ (95\% CI, 0.0-3.9\%) for single nitazoxanide. Overall CRs (including the placebo treatment arm), according to our 'gold' standard, were significantly lower compared to all diagnostic tests relying on a single stool sample ('gold' $7.2 \%$; single Kato-Katz, $16.4 \%$; duplicate Kato-Katz, $15.8 \%$; ether-concentration, $17.2 \%$; 'gold' vs. single Kato Katz $p<0.001$; 'gold' vs. duplicate Kato-Katz, $p$ $<0.001$; 'gold' vs. ether-concentration $p<0.001)$. Borderline significance was observed for quadruplicate Kato-Katz thick smears (CR: $10.8 \%$ ) compared to the 'gold' standard ( $p=$ 0.056). CRs based on quadruplicate Kato-Katz thick smears were significantly lower compared to diagnostic approaches based on a single stool sample (quadruplicate Kato-Katz vs. single Kato-Katz, $p=0.012$; duplicate Kato-Katz vs. single Kato-Katz, $p=0.035$; quadruplicate Kato-Katz vs. etherconcentration, $p=0.008$ ).

The overall CR according to the 'gold' standard against hookworm was $59.3 \%$ (95\% CI, 46.4-72.2\%). The CR for the individual diagnostic approaches were higher (Table 4); however, the differences lacked statistical significance. For
A. lumbricoides, we determined an overall CR of $63.9 \%$ (95\% CI, 47.4-80.4\%) using the 'gold' standard. Using single or duplicate Kato-Katz from a single stool sample and the ether-concentration technique resulted in lower overall CRs than the 'gold' standard, while higher CRs were recorded when analysing quadruplicate Kato-Katz (all $p>0.05$ ).

\section{Discussion}

Accurate diagnostic methods are mandatory for assessing soiltransmitted helminth infections, drug efficacies and the possible development of drug resistance [3, 5, 6, 27]. The most widely used technique for diagnosing soil-transmitted helminth infections is the Kato-Katz method [18]. Because of the low sensitivity of a single Kato-Katz thick smear, it is recommended to collect consecutive stool samples that are subjected to multiple Kato-Katz thick smears to enhance the sensitivity [12, 14-16]. We assessed the diagnostic performance of single, duplicate (from a single stool sample) and quadruplicate Kato-Katz thick smears (from two samples) in the frame of a randomised controlled trial and compared the results with an ether-concentration technique using formalinfixed stool samples. The latter method is rarely used in settings where soil-transmitted helminths are endemic, although it is often used in reference laboratories in Europe [22].

Our results demonstrate that the ether-concentration method has a similar or even higher sensitivity for the diagnosis of all three soil-transmitted helminths than single or duplicate Kato-Katz prepared from a single stool sample. Hence, we 
Table 2 Agreement between ether-concentration and Kato-Katz (different sampling efforts) for the diagnosis of soil-transmitted helminths among school-aged children on Pemba Island, Tanzania, in mid-2011

\begin{tabular}{|c|c|c|c|c|c|c|c|c|c|c|c|c|c|}
\hline \multirow[t]{2}{*}{ Before treatment } & & \multicolumn{2}{|c|}{$\begin{array}{l}\text { Ether- } \\
\text { concentration }\end{array}$} & \multirow[t]{2}{*}{ Total } & \multirow[t]{2}{*}{ Kappa } & \multirow[t]{2}{*}{ McNemar } & \multirow{2}{*}{\multicolumn{2}{|c|}{ After treatment }} & \multicolumn{2}{|c|}{$\begin{array}{l}\text { Ether- } \\
\text { concentration }\end{array}$} & \multirow[t]{2}{*}{ Total } & \multirow[t]{2}{*}{ Kappa } & \multirow[t]{2}{*}{ McNemar } \\
\hline & & - & + & & & & & & - & + & & & \\
\hline \multicolumn{14}{|l|}{ T. trichiura } \\
\hline \multirow[t]{3}{*}{ Single Kato-Katz } & - & 83 & 54 & 137 & 0.53 & $p=0.095$ & Single Kato-Katz & - & 101 & 44 & 145 & 0.55 & $p=0.361$ \\
\hline & + & 38 & 353 & 391 & & & & + & 53 & 330 & 383 & & \\
\hline & Total & 121 & 407 & 528 & & & & Total & 154 & 374 & 528 & & \\
\hline \multirow[t]{3}{*}{ Duplicate Kato-Katz } & - & 74 & 48 & 122 & 0.49 & $p=0.918$ & Duplicate Kato-Katz & - & 99 & 43 & 142 & 0.54 & $p=0.225$ \\
\hline & + & 47 & 359 & 406 & & & & + & 55 & 331 & 386 & & \\
\hline & Total & 121 & 407 & 528 & & & & Total & 154 & 374 & 528 & & \\
\hline \multirow{3}{*}{$\begin{array}{l}\text { Quadruplicate } \\
\text { Kato-Katz }\end{array}$} & - & 68 & 26 & 94 & 0.54 & $p=0.002$ & Quadruplicate & - & 80 & 28 & 108 & 0.49 & $p<0.001$ \\
\hline & + & 53 & 381 & 434 & & & Kato-Katz & + & 74 & 346 & 420 & & \\
\hline & Total & 121 & 407 & 528 & & & & Total & 154 & 374 & 528 & & \\
\hline \multicolumn{14}{|l|}{ Hookworm } \\
\hline \multirow[t]{3}{*}{ Single Kato-Katz } & - & 476 & 29 & 505 & 0.34 & $p=0.004$ & Single Kato-Katz & - & 495 & 14 & 509 & 0.37 & $p=0.549$ \\
\hline & + & 11 & 12 & 23 & & & & + & 11 & 8 & 19 & & \\
\hline & Total & 487 & 41 & 528 & & & & Total & 506 & 22 & 528 & & \\
\hline \multirow[t]{3}{*}{ Duplicate Kato-Katz } & - & 476 & 28 & 504 & 0.36 & $p=0.007$ & Duplicate Kato-Katz & - & 495 & 14 & 509 & 0.31 & $p=0.549$ \\
\hline & + & 11 & 13 & 24 & & & & + & 11 & 8 & 19 & & \\
\hline & Total & 487 & 41 & 528 & & & & Total & 506 & 22 & 528 & & \\
\hline \multirow{3}{*}{$\begin{array}{c}\text { Quadruplicate } \\
\text { Kato-Katz }\end{array}$} & - & 469 & 21 & 490 & 0.47 & $p=0.631$ & Quadruplicate & - & 490 & 11 & 501 & 0.43 & $p=0.336$ \\
\hline & + & 18 & 20 & 38 & & & Kato-Katz & + & 16 & 11 & 27 & & \\
\hline & Total & 487 & 41 & 528 & & & & Total & 506 & 22 & 528 & & \\
\hline \multicolumn{14}{|l|}{ A. lumbricoides } \\
\hline \multirow[t]{3}{*}{ Single Kato-Katz } & - & 504 & 10 & 514 & 0.62 & $p=0.092 *$ & Single Kato-Katz & - & 499 & 17 & 516 & 0.46 & $p=0.003 *$ \\
\hline & + & 3 & 11 & 14 & & & & + & 3 & 9 & 12 & & \\
\hline & Total & 507 & 21 & 528 & & & & Total & 502 & 26 & 528 & & \\
\hline \multirow[t]{3}{*}{ Duplicate Kato-Katz } & - & 502 & 10 & 512 & 0.58 & $p=0.302 *$ & Duplicate Kato-Katz & - & 499 & 17 & 516 & 0.46 & $p=0.003^{*}$ \\
\hline & + & 5 & 11 & 16 & & & & + & 3 & 9 & 12 & & \\
\hline & Total & 507 & 21 & 528 & & & & Total & 502 & 26 & 528 & & \\
\hline \multirow{3}{*}{$\begin{array}{c}\text { Quadruplicate } \\
\text { Kato-Katz }\end{array}$} & - & 492 & 9 & 501 & 0.48 & $p=0.221$ & Quadruplicate & - & 498 & 16 & 514 & 0.48 & $p=0.012 *$ \\
\hline & + & 15 & 12 & 27 & & & Kato-Katz & + & 4 & 10 & 14 & & \\
\hline & Total & 507 & 21 & 528 & & & & Total & 502 & 26 & 528 & & \\
\hline
\end{tabular}

*Instead of the McNemar $\chi^{2}$ value, the exact McNemar value was taken because a sample size of 20 individuals or less was in the discordant pair

speculate that a diagnostic examination based on two stool samples using the ether-concentration technique reveals a higher sensitivity than quadruplicate Kato-Katz thick smears. For diagnosing A. lumbricoides, the ether-concentration method showed a significantly higher sensitivity compared to quadruplicate Kato-Katz thick smears at the 3-week posttreatment follow-up. This might be explained by the large amount of stool $(\sim 2 \mathrm{~g})$ examined with the etherconcentration method, which should allow the detection of low infection intensities. The ether-concentration method offers the advantage of preservation. Stool samples are maintained in low-concentration formalin or within sodium acetate-acetic acid formalin (SAF), and, hence, can be kept for several weeks or months prior to microscopic examination for soil-transmitted helminth eggs as well as for intestinal protozoan cysts at a later time point [21,28]. This diagnostic technique is, therefore, a useful alternative to the Kato-Katz method, particularly in remote resource-constrained settings [29]. It is interesting to note that mobile phone microscopy might be an alternative promising diagnostic method in underserviced rural areas. The proof-of-concept of a 'firstgeneration' mobile phone light microscope was recently demonstrated in our study setting on Pemba Island [30].

The diagnostic accuracy was the same if either single or duplicate Kato-Katz thick smears were examined (single Kato-Katz vs. duplicate Kato-Katz). This result is in contrast to a recent study, which reported that multiple Kato-Katz thick smears from a single stool sample enhances the overall sensitivity for hookworm and T. trichiura [16]. Setting-specific differences in prevalence and infection intensities might 
Table 3 Prevalence of soil-transmitted helminth infections on Pemba Island, Tanzania, in mid-2011, according to different diagnostic methods and their corresponding sensitivities and negative predictive values 3 weeks post-treatment

\begin{tabular}{|c|c|c|c|c|c|c|}
\hline \multirow[t]{2}{*}{ Parasite } & \multirow[t]{2}{*}{ Diagnostic technique } & \multicolumn{3}{|c|}{ Number of infected school children } & \multirow[t]{2}{*}{ Sensitivity in $\%(95 \% \mathrm{CI})$} & \multirow[t]{2}{*}{$\mathrm{NPV}(95 \% \mathrm{CI})$} \\
\hline & & $n$ & $\%$ & $95 \% \mathrm{CI}$ & & \\
\hline \multirow[t]{5}{*}{ T. trichiura } & 'Gold' standard & 448 & 84.9 & $(81.8-87.9)$ & 100 & 100 \\
\hline & Single Kato-Katz & 383 & 72.5 & $(68.7-76.4)$ & $85.5(81.9-88.6)$ & $55.2(46.7-63.4)$ \\
\hline & Duplicate Kato-Katz & 386 & 73.1 & $(69.3-76.9)$ & $86.2(82.6-89.2)$ & $56.3(47.8-64.6)$ \\
\hline & Quadruplicate Kato-Katz & 420 & 79.6 & $(76.1-83.0)$ & $93.8(91.1-95.8)$ & $74.1(64.6-81.8)$ \\
\hline & Ether-concentration & 374 & 70.8 & $(66.9-74.7)$ & $83.5(79.7-86.8)$ & $51.9(43.8-60.0)$ \\
\hline \multirow[t]{5}{*}{ Hookworm } & 'Gold' standard & 38 & 7.2 & $(5.0-9.4)$ & 100 & 100 \\
\hline & Single Kato-Katz & 19 & 3.6 & $(2.0-5.2)$ & $50.0(33.4-66.6)$ & $96.3(94.1-97.7)$ \\
\hline & Duplicate Kato-Katz & 19 & 3.6 & $(2.0-5.2)$ & $50.0(33.4-66.6)$ & $96.3(94.1-97.7)$ \\
\hline & Quadruplicate Kato-Katz & 27 & 5.1 & $(3.2-7.0)$ & $71.1(54.1-84.6)$ & $97.8(96.0-98.8)$ \\
\hline & Ether-concentration & 22 & 4.2 & $(2.5-5.9)$ & $57.9(40.8-73.7)$ & $96.8(94.8-98.1)$ \\
\hline \multirow[t]{5}{*}{ A. lumbricoides } & 'Gold' standard & 30 & 5.7 & $(3.7-7.7)$ & 100 & 100 \\
\hline & Single Kato-Katz & 12 & 2.3 & $(1.0-3.6)$ & $40.0(22.7-59.4)$ & $96.5(94.4-97.9)$ \\
\hline & Duplicate Kato-Katz & 12 & 2.3 & $(1.0-3.6)$ & $40.0(22.7-59.4)$ & $96.5(94.4-97.9)$ \\
\hline & Quadruplicate Kato-Katz & 14 & 2.7 & $(1.3-4.0)$ & $46.7(28.3-65.7)$ & $96.9(94.9-98.1)$ \\
\hline & Ether-concentration & 26 & 4.9 & $(3.1-6.8)$ & $86.7(69.3-96.2)$ & $99.2(97.8-99.7)$ \\
\hline
\end{tabular}

explain this observation $[8,31-33]$. On the other hand, collecting multiple stool samples had a significant effect on improving the diagnostic sensitivity, and this observation corroborates previous findings [12, 14-16]. Hence, collecting multiple stool samples is often the method of choice for diagnosing soil-transmitted helminths in intervention trials.

Table 4 Cure rates (CR) determined following treatment with nitazoxanide-albendazole, albendazole, nitazoxanide and placebo against soil-transmitted helminths among school-aged children on Pemba Island, Tanzania, according to different diagnostic techniques

\begin{tabular}{|c|c|c|c|c|c|}
\hline & \multicolumn{5}{|l|}{ Cure rate } \\
\hline & Overall & Nitazoxanide-albendazole & Albendazole & Nitazoxanide & Placebo \\
\hline \multicolumn{6}{|l|}{ T. trichiura } \\
\hline Single Kato-Katz (95 \% CI) & $16.4(12.7-20.1)$ & $19.8(11.2-28.4)$ & $20.4(12.3-28.5)$ & $12.9(6.2-19.5)$ & $13.2(6.7-19.8)$ \\
\hline Duplicate Kato-Katz (95 \% CI) & $15.8(12.2-19.3)$ & $19.3(10.9-27.7)$ & $19.8(11.9-27.7)$ & $11.2(5.1-17.3)$ & $13.6(7.1-20.2)$ \\
\hline Quadruplicate Kato-Katz (95\% CI) & $10.8(7.9-13.8)$ & $14.6(7.4-21.8)$ & $15.2(8.2-22.2)$ & $5.3(1.1-9.4)$ & $9.2(4.0-14.5)$ \\
\hline Ether-concentration $(95 \% \mathrm{CI})$ & $17.2(13.5-20.9)$ & $22.0(13.3-30.6)$ & $17.3(9.7-25.0)$ & $19.4(11.9-27.0)$ & $10.9(5.0-16.8)$ \\
\hline 'Gold' standard & $7.2(4.8-9.5)$ & $11.7(5.3-18.0)$ & $9.1(3.6-14.5)$ & $1.6(0.0-3.9)$ & $7.2(2.6-11.8)$ \\
\hline \multicolumn{6}{|l|}{ Hookworm } \\
\hline Single Kato-Katz (95 \% CI) & $69.6(49.2-89.9)$ & $80.0(24.5-100.0)$ & $83.3(40.5-100.0)$ & $83.3(40.5-100.0)$ & $33.3(0.0-87.5)$ \\
\hline Duplicate Kato-Katz (95 \% CI) & $66.7(46.3-87.0)$ & $80.0(24.5-100.0)$ & $83.3(40.5-100.0)$ & $71.4(26.3-100.0)$ & $33.3(0.0-87.5)$ \\
\hline Quadruplicate Kato-Katz (95\% CI) & $71.1(55.9-86.2)$ & $88.9(63.3-100.0)$ & $80.0(49.8-100.0)$ & $66.7(28.2-100.0)$ & $50.0(12.3-87.7)$ \\
\hline Ether-concentration $(95 \% \mathrm{CI})$ & $73.2(59.0-87.3)$ & $83.3(58.6-100.0)$ & $100.0(-)$ & $33.3(0.0-71.8)$ & $75.0(46.3-100.0)$ \\
\hline 'Gold' standard & $59.3(46.4-72.2)$ & $75.0(51.2-98.8)$ & $75.0(46.3-100.0)$ & $38.5(7.9-69.1)$ & $50.0(24.4-75.6)$ \\
\hline \multicolumn{6}{|l|}{ A. lumbricoides } \\
\hline Single Kato-Katz (95 \% CI) & $57.1(27.5-86.8)$ & $100.0(-)$ & $100.0(-)$ & $50.0(0.0-100.0)$ & $0.0(-)$ \\
\hline Duplicate Kato-Katz (95 \% CI) & $62.5(35.9-89.1)$ & $100.0(-)$ & $100.0(-)$ & $50.0(0.0-100.0)$ & $0.0(-)$ \\
\hline Quadruplicate Kato-Katz (95\% CI) & $70.4(52.0-88.8)$ & $100.0(-)$ & $100.0(-)$ & $70.0(35.4-100.0)$ & $16.7(0.0-59.5)$ \\
\hline Ether-concentration $(95 \% \mathrm{CI})$ & $52.4(29.1-75.7)$ & $100.0(-)$ & $100.0(-)$ & $50.0(12.3-87.7)$ & $0.0(-)$ \\
\hline 'Gold' standard & $63.9(47.4-80.4)$ & $100.0(-)$ & $87.5(57.9-100.0)$ & $60.0(31.9-88.1)$ & $14.3(0.0-49.2)$ \\
\hline
\end{tabular}


We observed significantly lower CRs for T. trichiura, preparing duplicate Kato-Katz thick smears from two stool samples compared to all diagnostic methods relying on only one stool sample, which re-emphasises the importance of an accurate diagnostic test in clinical trials. Further, our 'gold' standard had lower CRs compared to quadruplicate Kato-Katz thick smears for T. trichiura. Even though this result showed borderline significance, it demonstrates that sensitive tools for diagnosing soil-transmitted helminth infections are warranted. On the other hand, it is interesting to note that duplicate Kato-Katz revealed higher CRs for $A$. lumbricoides. However, no statistical significance was observed, but this might be explained by the relatively small sample size. Several studies showed that the FLOTAC technique revealed higher sensitivities compared to multiple stool samples examined with the Kato-Katz method $[15,16,32,34-36]$ and also compared to the etherconcentration technique [16]. However, the FLOTAC technique, as well as the ether-concentration method, requires additional laboratory equipment, such as a centrifuge, and is also more expensive than the Kato-Katz method [10].

Our study has the following limitations. First and foremost, for the ether-concentration method, helminth eggs were only counted in a semi-quantitative manner. Therefore, it was only possible to calculate the prevalence and $\mathrm{CR}$, but no data could be provided on infection intensities and egg reduction rates, an important parameter for the evaluation of anthelminthic drug efficacy [37]. Second, all positive results obtained by the different techniques were recorded as "true positive". Though this is a common standard in helminth diagnosis $[33,38]$, it is worth highlighting that false-positive results might be reported even by well-trained laboratory technicians due to artefacts in stool that are misinterpreted as helminth eggs or due to writing errors on the entry forms. To our knowledge, the effect of false-positive results for the diagnosis of soil-transmitted helminths has not yet been studied. Third, all diagnostic approaches revealed high CRs against hookworm in the placebo group (as high as $75 \%$ for the ether-concentration technique), indicating a diagnostic problem for hookworm in general. We have previously attributed this finding to the low prevalence and intensity of hookworm infections [20]. Finally, as mentioned before, the sample size for hookworm as well as for A. lumbricoides was relatively small due to the low prevalence of these infections in our study setting (11.2\% and $6.8 \%$ at baseline, respectively). Therefore, even though we did not find significant differences between the diagnostic techniques for these two helminth species, we should not assume that they do not exist. Note that, also, the sensitivities and NPVs are strongly influenced by prevalence [5]. Thus, these parameters should only be compared among different diagnostic methods and not among helminth species. A negative diagnostic result is more likely to be true (high NPV) when the prevalence is low, as was the case in our study for hookworm and A. lumbricoides.
To conclude, our study confirmed that a sensitive diagnostic method is crucial in order to reliably assess the prevalence of soil-transmitted helminth infections, as well as determine CRs in clinical trials, and that using insensitive diagnostic methods overestimates drug efficacy when CR is employed as the outcome measure. Our trial re-emphasises that collecting multiple stool samples is useful to enhance the sensitivity, especially in settings where infection intensities are low. The diagnostic accuracy of the ether-concentration method from a single formalin-fixed stool sample revealed moderate diagnostic agreement with quadruplicate Kato-Katz thick smears and a similar or even higher sensitivity for the diagnosis of all three soil-transmitted helminths than single or duplicate Kato-Katz thick smears prepared from a single stool sample. Hence, the ether-concentration method provides an alternative in settings where fresh stool samples cannot be directly examined.

Acknowledgements We are grateful to the children attending Wawi and Al-Sadik schools for providing the stool samples. We express our thanks to the whole team from the Public Health Laboratory-Ivo de Carneri, on Pemba Island, Tanzania, for their work in the field and in the laboratory, and to the team from the Department of Medical and Diagnostic Services at the Swiss Tropical and Public Health Institute. This trial was financially supported by the Medicor Foundation and the University of Basel. JU acknowledges financial support by the Swiss National Science Foundation (SNSF, project no. 320030_141246). JK is grateful to the SNSF for a personal career development grant (project nos. PPOOA-114941 and PPOOP3_135170).

Conflict of interest The authors declare that they have no conflict of interest.

\section{References}

1. Murray CJL, Vos T, Lozano R, Naghavi M, Flaxman AD, Michaud C et al (2012) Disability-adjusted life years (DALYs) for 291 diseases and injuries in 21 regions, 1990-2010: a systematic analysis for the Global Burden of Disease Study 2010. Lancet 380:2197-2223

2. World Health Organization (2006) Preventive chemotherapy in human helminthiasis. Coordinated use of anthelminthic drugs in control interventions: a manual for health professionals and programme managers. WHO, Geneva, pp 1-62

3. Hotez PJ, Molyneux DH, Fenwick A, Kumaresan J, Ehrlich Sachs S, Sachs JD et al (2007) Control of neglected tropical diseases. N Engl J Med 357:1018-1027

4. World Health Organization (2013) Sustaining the drive to overcome the global impact of neglected tropical diseases. Second WHO report on neglected tropical diseases. WHO, Geneva, pp 1-139

5. Bergquist R, Johansen MV, Utzinger J (2009) Diagnostic dilemmas in helminthology: what tools to use and when? Trends Parasitol 25: 151-156

6. McCarthy JS, Lustigman S, Yang GJ, Barakat RM, García HH, Sripa B et al (2012) A research agenda for helminth diseases of humans: diagnostics for control and elimination programmes. PLoS Negl Trop Dis 6:e1601 
7. Katz N, Chaves A, Pellegrino J (1972) A simple device for quantitative stool thick-smear technique in schistosomiasis mansoni. Rev Inst Med Trop São Paulo 14:397-400

8. Booth M, Vounatsou P, N'Goran EK, Tanner M, Utzinger J (2003) The influence of sampling effort and the performance of the KatoKatz technique in diagnosing Schistosoma mansoni and hookworm co-infections in rural Côte d'Ivoire. Parasitology 127:525-531

9. Lin D-D, Liu J-X, Liu Y-M, Hu F, Zhang Y-Y, Xu J-M et al (2008) Routine Kato-Katz technique underestimates the prevalence of Schistosoma japonicum: a case study in an endemic area of the People's Republic of China. Parasitol Int 57:281-286

10. Speich B, Knopp S, Mohammed KA, Khamis IS, Rinaldi L, Cringoli $\mathrm{G}$ et al (2010) Comparative cost assessment of the Kato-Katz and FLOTAC techniques for soil-transmitted helminth diagnosis in epidemiological surveys. Parasit Vectors 3:71

11. Lovis L, Mak TK, Phongluxa K, Ayé Soukhathammavong P, Vonghachack Y, Keiser J et al (2012) Efficacy of praziquantel against Schistosoma mekongi and Opisthorchis viverrini: a randomized, single-blinded dose-comparison trial. PLoS Negl Trop Dis 6:e1726

12. Ebrahim A, El-Morshedy H, Omer E, El-Daly S, Barakat R (1997) Evaluation of the Kato-Katz thick smear and formol ether sedimentation techniques for quantitative diagnosis of Schistosoma mansoni infection. Am J Trop Med Hyg 57:706-708

13. Utzinger J, Booth M, N'Goran EK, Müller I, Tanner M, Lengeler C (2001) Relative contribution of day-to-day and intra-specimen variation in faecal egg counts of Schistosoma mansoni before and after treatment with praziquantel. Parasitology 122:537-544

14. Steinmann P, Du ZW, Wang LB, Wang XZ, Jiang JY, Li LH et al (2008) Extensive multiparasitism in a village of Yunnan province, People's Republic of China, revealed by a suite of diagnostic methods. Am J Trop Med Hyg 78:760-769

15. Knopp S, Rinaldi L, Khamis IS, Stothard JR, Rollinson D, Maurelli MP et al (2009) A single FLOTAC is more sensitive than triplicate Kato-Katz for the diagnosis of low-intensity soil-transmitted helminth infections. Trans R Soc Trop Med Hyg 103:347-354

16. Glinz D, Silué KD, Knopp S, Lohourignon LK, Yao KP, Steinmann P et al (2010) Comparing diagnostic accuracy of Kato-Katz, Koga agar plate, ether-concentration, and FLOTAC for Schistosoma mansoni and soil-transmitted helminths. PLoS Negl Trop Dis 4:e754

17. Levallois $P$, Chevalier P, Gingras S, Déry P, Payment P, Michel P et al (2013) Risk of infectious gastroenteritis in young children living in Québec rural areas with intensive animal farming: results of a casecontrol study (2004-2007). Zoonoses Public Health. doi:10.1111/ zph.12039

18. World Health Organization (2008) Action against worms. February 2008, Issue 11

19. Allen AVH, Ridley DS (1970) Further observations on the formolether concentration technique for faecal parasites. J Clin Pathol 23: 545-546

20. Speich B, Ame SM, Ali SM, Alles R, Hattendorf J, Utzinger J et al (2012) Efficacy and safety of nitazoxanide, albendazole, and nitazoxanide-albendazole against Trichuris trichiura infection: a randomized controlled trial. PLoS Negl Trop Dis 6:e1685

21. Marti H, Escher E (1990) SAF - an alternative fixation solution for parasitological stool specimens. Schweiz Med Wochenschr 120: $1473-1476$

22. Utzinger J, Botero-Kleiven S, Castelli F, Chiodini PL, Edwards H, Köhler N et al (2010) Microscopic diagnosis of sodium acetate-acetic acid-formalin-fixed stool samples for helminths and intestinal protozoa: a comparison among European reference laboratories. Clin Microbiol Infect 16:267-273

23. Speich B, Marti H, Ame SM, Ali SM, Bogoch II, Utzinger J et al (2013) Prevalence of intestinal protozoa infection among school-aged children on Pemba Island, Tanzania, and effect of single-dose albendazole, nitazoxanide and albendazole-nitazoxanide. Parasit Vectors 6:3

24. Cohen J (1960) A coefficient of agreement for nominal scales. Educ Psychol Meas 20:37-46

25. Landis JR, Koch GG (1977) The measurement of observer agreement for categorical data. Biometrics 33:159-174

26. Hawass NE (1997) Comparing the sensitivities and specificities of two diagnostic procedures performed on the same group of patients. Br J Radiol 70:360-366

27. Albonico M, Engels D, Savioli L (2004) Monitoring drug efficacy and early detection of drug resistance in human soil-transmitted nematodes: a pressing public health agenda for helminth control. Int J Parasitol 34:1205-1210

28. Cringoli G, Rinaldi L, Maurelli MP, Utzinger J (2010) FLOTAC: new multivalent techniques for qualitative and quantitative copromicroscopic diagnosis of parasites in animals and humans. Nat Protoc 5:503-515

29. King JD, Endeshaw T, Escher E, Alemtaye G, Melaku S, Gelaye W et al (2013) Intestinal parasite prevalence in an area of Ethiopia after implementing the SAFE strategy, enhanced outreach services, and health extension program. PLoS Negl Trop Dis 7:e2223

30. Bogoch II, Andrews JR, Speich B, Utzinger J, Ame SM, Ali SM et al (2013) Mobile phone microscopy for the diagnosis of soiltransmitted helminth infections: a proof-of-concept study. Am J Trop Med Hyg 88:626-629

31. Khieu V, Schär F, Marti H, Sayasone S, Duong S, Muth S et al (2013) Diagnosis, treatment and risk factors of Strongyloides stercoralis in schoolchildren in Cambodia. PLoS Negl Trop Dis 7:e2035

32. Jeandron A, Abdyldaieva G, Usubalieva J, Ensink JH, Cox J, Matthys B et al (2010) Accuracy of the Kato-Katz, adhesive tape and FLOTAC techniques for helminth diagnosis among children in Kyrgyzstan. Acta Trop 116:185-192

33. Knopp S, Mgeni AF, Khamis IS, Steinmann P, Stothard JR, Rollinson D et al (2008) Diagnosis of soil-transmitted helminths in the era of preventive chemotherapy: effect of multiple stool sampling and use of different diagnostic techniques. PLoS Negl Trop Dis 2:e331

34. Utzinger J, Rinaldi L, Lohourignon LK, Rohner F, Zimmermann $\mathrm{MB}$, Tschannen AB et al (2008) FLOTAC: a new sensitive technique for the diagnosis of hookworm infections in humans. Trans R Soc Trop Med Hyg 102:84-90

35. Habtamu K, Degarege A, Ye-Ebiyo Y, Erko B (2011) Comparison of the Kato-Katz and FLOTAC techniques for the diagnosis of soiltransmitted helminth infections. Parasitol Int 60:398-402

36. Knopp S, Speich B, Hattendorf J, Rinaldi L, Mohammed KA, Khamis IS et al (2011) Diagnostic accuracy of Kato-Katz and FLOTAC for assessing anthelmintic drug efficacy. PLoS Negl Trop Dis 5:e1036

37. World Health Organization (2008) Working group on soiltransmitted helminthiasis. Monitoring anthelmintic efficacy for soil transmitted helminths $(\mathrm{STH})$

38. Bogoch II, Raso G, N'Goran EK, Marti HP, Utzinger J (2006) Differences in microscopic diagnosis of helminths and intestinal protozoa among diagnostic centres. Eur J Clin Microbiol Infect Dis $25: 344-347$ 